\title{
Quality Assurance on the welding work during the assembly of Wendelstein 7-X
}

\author{
Michael Schröder, W7-X Team \\ Max-Planck-Institut für Plasmaphysik, Greifswald, Germany
}

\begin{abstract}
At the Max-Planck-Institut für Plasmaphysik (IPP) in Greifswald (Germany) the stellarator experiment W7-X is presently being assembled. During this assembly many different weld connections are made, which are very important for the proper functionality of the experiment. This concerns mainly the structural integrity and the leak tightness. The quality requirements for the weld seams are high (mainly class B according to DIN EN ISO 5817), because the complex machine must operate reliably for more than 15 years and the possibility of any repair or change of important components is very small. To guarantee a high quality of the welds they are submitted to different tests. The applied test methods are depending on the function of the weld, the wall thickness, the seam geometry, and the material. The main test methods are visual testing (VT), penetrant testing (PT), radiographic testing (RT), ultrasonic testing (UT), leak testing (LT), permeability testing and macros. The paper will describe the application of these test methods and show their need by examples of typical weld imperfections.
\end{abstract}

Keywords: quality assurance, welding, testing, NDT

\section{Introduction}

Welding is one of the essential processes during the assembly of W7-X. Beside welds with subordinated significance there are many welds which have an essential importance for the functionality of the experiment. It must be made sure that these weld seams can either sustain the mechanical loads or keep their leak tightness during the whole time of operation (15 y). The different weld testing methods assure the weld quality and are similarly the base for the detection and correction of defects. Simultaneously they support the raising of the quality awareness in the project. In the following some important welds of W7-X and their tests are described.

\section{Cryostat welds}

\subsection{General}

The cryostat which surrounds the superconducting magnet coils consists of the plasma vessel, the outer vessel with domes, and the ports. All these components are welded structures and are connected with each other by TIG welding. The welds have to be stable and leak tight. The material of all components is austenitic steel 1.4429. The rules and standards for the manufacture and tests are AD2000. All welds are qualified by welding procedure qualifications (WPQ). (Figures and further information on the cryostat: [1, 2, 3])

\subsection{Plasma vessel (PV)}

The PV consists of 20 prefabricated, all welded segments (4 per module). The wall thickness is $17 \mathrm{~mm}$. All welds are girth welds with full penetration. The weld shape is $\mathrm{V}$ or $\mathrm{X}$ according to accessibility from one or from both sides. All assembly and the most test works are done by MAN Diesel \& Turbo Deggendorf (is valid also for 2.3 and 2.4).

The following weld tests are carried out:
- VT of all passes (root, intermediate, final)

- $\quad$ PT of the root and the final pass

- RT 100\%, if accessible

- UT $100 \%$, if accessible

- $\operatorname{LT} 100 \%$

- Permeability tests $10 \%$ of the weld length

The most critical and complicated part of the weld is the root, therefore the PT application there. But it is important to remove the penetrant residue to avoid pores and lack of fusion in the following passes. The large opening angle of the weld and the smooth surface because of the TIG welding allows here an efficient cleaning of the penetrant residue. Also with RT it is possible to find root imperfections. At V-welds here the significance of UT is limited.

The weld volume is tested by UT. For the evaluation the distance amplitude correction curve (DAC) method is used. The DGS method (Distance Gain Size) is not usable because of the material. The calibration of the test equipment with special probes for austenitic welds is carried out on special reference blocks for every weld form. For this special UT experience is necessary. Therefore the tests are carried out by an expert inspector from MAN, not by a normal service company.

\subsection{Outer vessel (OV)}

The OV consists of 5 modules and each of a lower and an upper shell (wall thickness $25 \mathrm{~mm}$ ). All welds are full penetrating V-welds, which are accessible for welding and testing only from the outside. The same tests are used as in 2.2 except LT. The RT evaluation is limited because of a backing strip and has to be done by expert personnel.

\subsection{Dome - Outer Vessel welds}

During assembly all domes which are located in the separation plane between lower and upper shell of the 
OV have to be welded with the OV. These welds are normally not full penetrated and are only accessible from the outside. In this case the following weld tests are carried out:

- $\quad$ VT of all passes (root, intermediate, final)

- $\quad$ PT of final pass

- $\quad$ UT (limited significance)

- Permeability tests

\subsection{Port - plasma Vessel welds}

The welds between ports and PV are not full penetrated (HU or fillet welds) and only accessible from the inside of the PV. The ports are presently assembled and the welding in the first module will start in autumn 2010. The welding and testing will be done by IPP personnel. Intended tests are:

- $\quad$ VT of all passes (root, intermediate, final)

- $\quad$ PT (under discussion)

- Permeability tests

The scope of PT is presently under discussion because of two points: Because RT and UT are not possible, it is intended to test all passes by PT but because of the narrow weld gap (HU weld) it is feared that penetrant residue involve pores and lack of fusion in the following passes. Furthermore unfavorable effects from the penetrant residue for the ultra high vacuum in the PV are feared.

\subsection{Test Results}

At the cryostat welds imperfections were hardly detected. A reason for it is certainly the high level of testing which encouraged the welders always to produce high quality.

\section{Welds in the magnet system}

\subsection{General}

The magnet system consists of 50 non-planar (10 per module) and 20 planar coils (4 per module), which are screwed with an inner central support structure. On the outside the non-planar coils within each module are welded together with so-called Lateral Support Elements (LSE) which are clamped between pressure supports of the the slightly forced apart coils (Fig. 1, 2). The LSE are made with a defined oversize, so that after the welding because of the shrinking the coils are in the required position.

\subsection{Lateral Support Elements (LSE) welds}

The function of the LSE welds is a structuralmechanical one, because the Lorentz forces on the coils must be transferred by these elements to form a "supporting shell" of the magnet system on the outside. The coil casings consist of austenitic steel casting (1.3960). The material of the LSE is 1.4429. The cast \# material has typical defects like particular shrinkage cavities and hot cracks. These defects can open up by the warm influence of the welding or their size can become critical (Fig. 2). Furthermore at some pressure supports former copper stripes for cooling had to be removed, because of a design change. The leftover of these copper welds causes cracks, which can also open during welding.

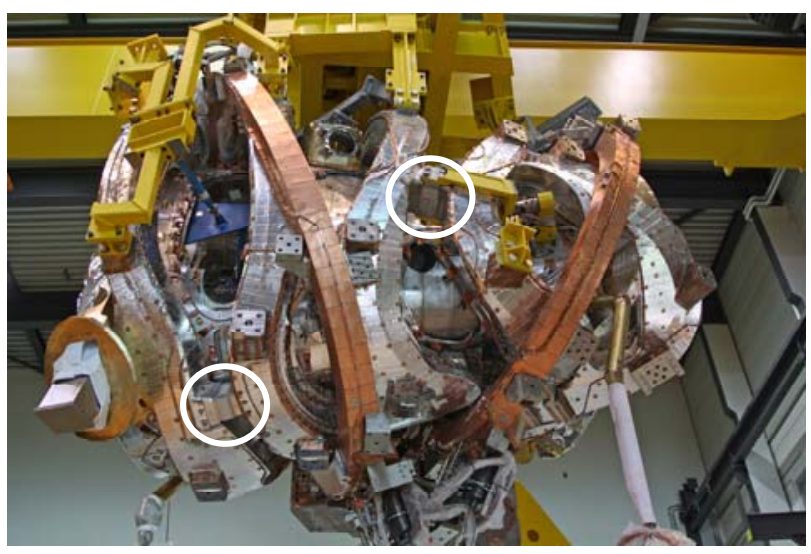

Fig.1 Configuration of LSE in the coil system (one half module)

For the welding process MAG welding was chosen to insert preferably few heat to keep the weld distortion low. This is necessary because the weld distortion influences the geometry of the magnet system. Partly the accessibility for the welder is difficult. For all this reasons there is especially a risk of lack of fusion and hot cracks.

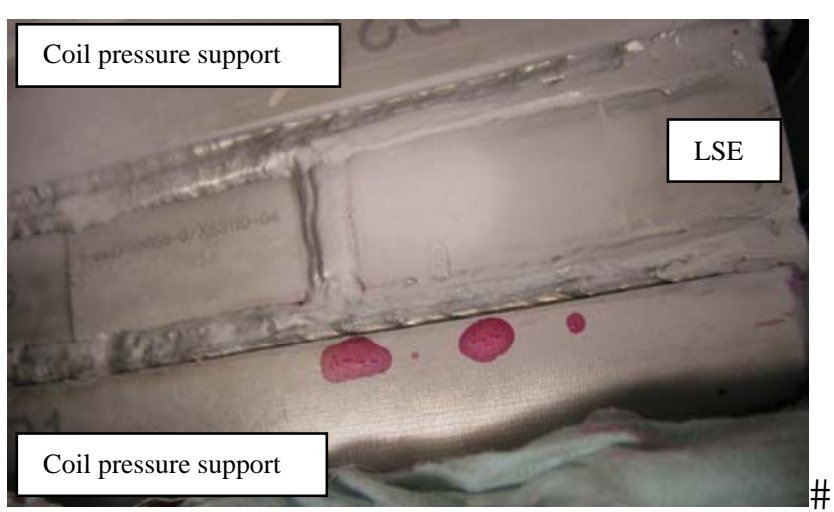

Fig.2 PT indications in the HAZ (1.3960) after LSE welding

For the evaluation of the weld and heat affected zone criteria had to be found because the coil casings were specified in accordance to DIN 1690-2 [4] class 3 respectively class 2 in welding areas and the acceptable imperfections in accordance of this standard are much larger than in common welding standards (e.g. DIN EN ISO 5817 [5]), which are used usually in the project. Therefore on the base of calculations limits for crack lengths were fixed. These allowable defects are significant greater than DIN EN 5817 allows.

The following tests are carried out:

- VT of all passes (root, intermediate, final)

- $\quad$ PT of final pass 
RT and UT are not possible because of the geometry. PT of root and intermediate passes was renounced because of the cleanness in the welding groove.

In August 2010 the last of 50 LSE was welded and tested. Most imperfections detected resulted from indications of the casting. With PT 2.5 times more unacceptable indications were found than with VT . This shows that it has made sense to apply this procedure here.

\subsection{Al welds on the superconductor}

Each group of ten equal coils is connected with the respective current feed-through by a superconducting bus system. The connections between the coils and the bus system are made with special transition pieces consisting of an Aluminium/Stainless Steel diffusion bonded piece. These pieces must be welded on one side to the aluminium jacket of the superconductor. Each of these all in all 382 welds has to be Helium leak tight for the whole period of the operation. Some aspects of the quality assurance in particular the weld qualification are described in [6]. In the meantime the transition pieces of all coils (140 pieces) and the major part of the bus system (144) are welded.

Because of the weld design only VT and LT as NDT methods can be carried out. Therefore, the quality assurance of these welds occurs, in addition, with the examination of working proof samples from which macros are made. Before every welding of a transition piece at a coil one working proof sample was welded and tested. In order to observe changes of the weld quality a gliding average of the length of cracks and amount of pores of the respectively last 10 samples was determined, (Fig. 3). The evaluation of these data was used to initiate countermeasures eventually. Thus it could be reached that pores which had led in the meantime to rejections were hardly relevant later on. However, hot cracks appeared over and over again. The attempt to minimize this by less welding heat had to be balanced with the risk of lower penetration and the lack of fusion which may reduce the stability against Helium leaks. Nevertheless the evaluation of all macros can only be a hint which imperfections may be present in the final real welds.

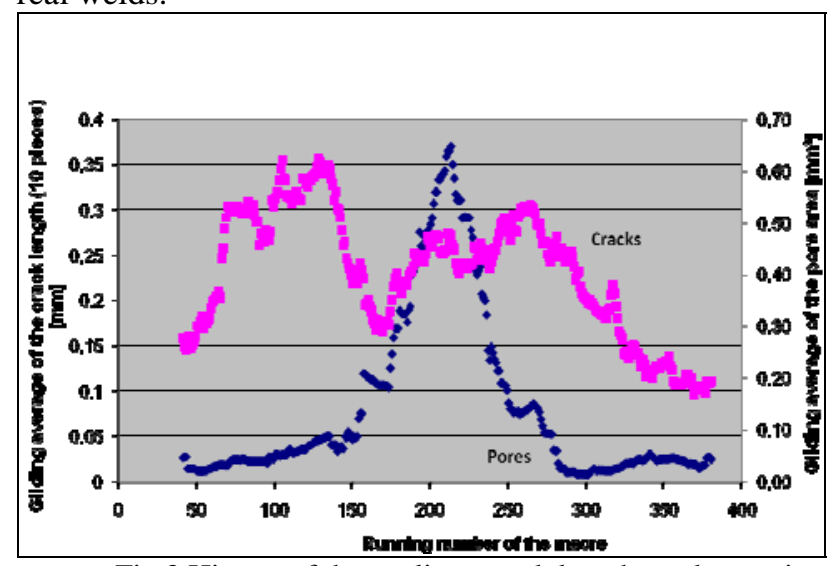

Fig.3 History of the quality - crack lengths and pores in Al-welds

\section{Pipe welds}

\subsection{General}

In W7-X there is a huge number of the pipes which are connected by welding with each other. These are:

- Cryo-pipes for the superconducting coil system (conductor cooling, cooling of the coil casings, the central support structure and the panels of the thermal insulation on the PV and OV). The medium in these pipes is $\mathrm{He}$ from room temperature to $4 \mathrm{~K}$

- Water pipes for cooling and bake-out of the PV and the ports and for the cooling of the components in the PV. The medium in these pipes is water from room temperature to $150^{\circ} \mathrm{C}$

All these pipes have a complicated 3D geometry, are mostly hardly accessible and are hardly repairable in the later operation (Fig. 4). The dimensions reach from $\varnothing 9 \times 1 \mathrm{~mm}$ to $\varnothing 42 \times 3 \mathrm{~mm}$. The welding process is TIG or TIG orbital. The welds are single-pass. As forming gas an Argon Hydrogen mixture is used. All welds have to be leak tight for 15 years operation and of course in the time up to the commissioning (in 2014). All welds are qualified by welding procedure qualifications (WPQ). While during the operation the inert gas helium is inside the cryo-pipes, they are exposed up to the commissioning to the environmental conditions of the assembly shop. Therefore, because of the risk of corrosion the avoidance of temper colours is important.

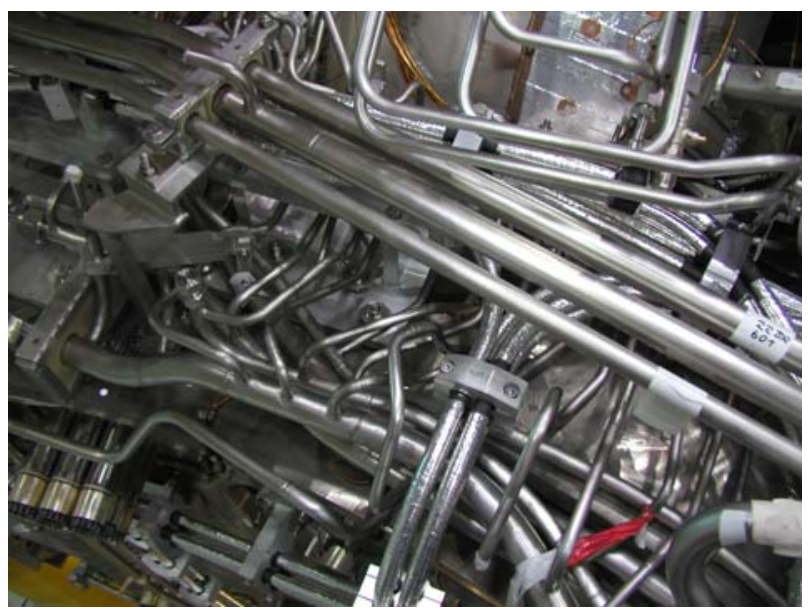

Fig.4 Impression about the assembly conditions of the cryo pipes

\subsection{Weld imperfections and testing}

Typical weld imperfections are root and outer surface imperfections, temper colours and inner volume imperfections as pores or tungsten inclusions. However, an examination of 100 assembly welds with RT showed that pores and tungsten inclusions appear only very seldom and in low size. They are assessed as less critical. Lack of side wall fusion which are not open to the root or the outer surface are not expected because of the small wall thickness. Furthermore the experience showed that handmade and orbital welds are equally susceptible to 
imperfections. With the orbital welding small differences of wall thicknesses, linear misalignment or different material charges may can induce root imperfections.

From this experience the following tests were made independently of being a handmade and an orbital weld:

- $\quad$ VT from outside $100 \%$

- VT from inside by videoscope $100 \%$ (if possible)

- $\quad \mathrm{RT}$, if videoscopy is not possible

- $\quad$ LT $100 \%$ (at cryo-pipes after quick-freeze by $\mathrm{LN}_{2}$ )

The acceptance criterion is class B of DIN EN ISO 5817. Only for orbital welds more incompletely fillet groove ( $0.2 \mathrm{x}$ wall thickness) is allowed to make sure that the root is full penetrated. Temper colours are not allowed.

For the inner VT stiff endoscopes are applicable only in special cases because of the complicated pipe geometry. Therefore different flexible videoscopes are available (probe diameter 3.9 and $6.1 \mathrm{~mm}$, probe length 3 and 6 $\mathrm{m})$. Every probe can be equipped with different frontand sideview object lenses. The videoscopy of a weld is only complete, if it is examined with both lenses. By videoscopy root imperfections and colours are visible. The test staff needs much experience to evaluate the indications, because of the magnification and the colour indication.

In generally RT is done in accordance to DIN EN 1435, class B [7], but often it was not possible to make the required number of partial exposures because of difficult accessibility.

\subsection{Test results}

The cryo-pipe welding and testing of the first 3 modules is finished. From 1278 weld seams (99 \% orbital welded) $40 \%$ could be tested from the inside by videoscopy. The repair ratio of the first modul was $4.3 \%$ but the ratio in the last two modules could be improved to an average of $2.6 \%$. The fraction of the different kinds of imperfections which led to repairs, shows Fig. 5. Because every imperfection can be a risk for the operation, a reduction of the test extent is not planned.

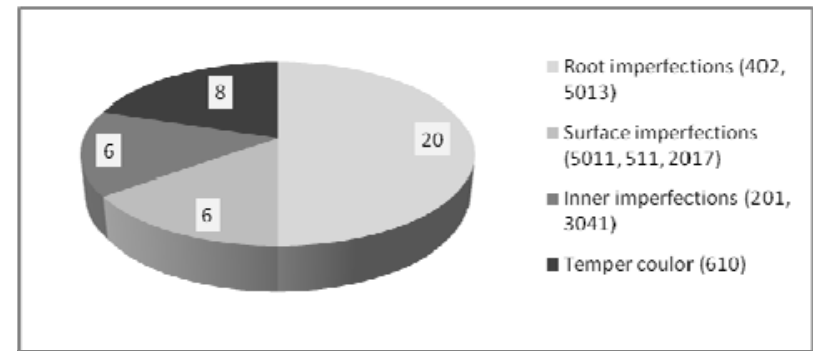

Fig.5 Imperfections founded in cryo pipe welds (Reference No. acc. DIN EN ISO 6520-1)

\section{Test staff}

It is aimed that all inspectors of the QM department who are carrying out NDT methods are certificated to DIN EN 473 [8]. For external Test personnel (e.g. RT) this is expressly required. Internal inspectors are appointed after general and special training [9]. Special experiences are necessary particularly for UT and VT (videoscopy).

\section{Conclusions}

The weld testings makes an essential contribution to the Quality assurance in the project and with it to a safe operation of W7-X.

The use of the different test methods requires trained and experienced staff, suitable test equipments, and well proven test procedures.

Some welds can only be tested in a restricted way because the design did not account for these tests or the geometrical situation has become more difficult as the assembly is more complete. A test-appropriate design of welded components is already necessary in the early design stage.

\section{Acknowledgments}

The work was done in close cooperation with the departments of W7-X. In particular the author would like to thank all members of the QM department for their help.

\section{References}

[1] B. Hein, Manufacturing and assembly of the plasma- and outer vessel of the cryostat for Wendelstein 7-X, this conference.

[2] T. Koppe, Overview of main-mechanical components and critical manufacturing aspects of the Wendelstein 7-X cryostat, this conference.

[3] D. Hartmann, Building a large cryogenic fusion device experience from $\mathrm{W} 7-\mathrm{X}$, this conference.

[4] DIN 1690-2: Technical delivery conditions for castings of metallic materials; classification oft he quality of steel castings on the basis of non destructive tests, 1985

[5] DIN EN ISO 5817: Welding - Fusion-welded joints in steel, nickel, titanium and their alloys (beam welding excluded) - Quality levels for imperfections; German version 2006

[6] M. Schröder et al., Quality assurance of aluminium weld seams and cast casings oft he W7-X coils

[7] DIN EN 1435: NDT of welds - Radiographic testing of welded joints; German version 2002

[8] DIN EN 473: Non-destructiv testing - Qualification and certification of NDT personnel - General principles; German version 2008

[9] R. Vilbrandt, Quality assurance during assembly of Wendelstein 7-X, this conference. 